EL ANTONIANO. 2020; 135: 3-17

Recepción: 05-08-2020

Aprobación: 15-11-2020

\title{
EFECTOS SOCIALES DEL PROCESO DE LICENCIAMIENTO UNIVERSITARIO EN LOS ESTUDIANTES DE UNIVERSIDADES NO LICENCIADAS EN LA REGIÓN DEL CUSCO
}

\section{SOCIAL EFFECTS OF THE LICENSING PROCESS ON STUDENTS FROM UNLICENSED UNIVERSITIES IN CUSCO}

Flórez Mujica; Indira Yesenia indira.florez@unsaac.edu.pe, https://orcid.org/0000-0002-3424-8739

\section{RESUMEN}

El artículo presenta el análisis descriptivo de los efectos sociales del proceso de Licenciamiento Universitario en los estudiantes de Universidades no licenciadas en la región del Cusco, bajo las categorias de deserción y migración educativa. La investigación es de enfoque mixto, nivel descriptivo y diseño no experimental - transversal. Se aplicó una encuesta a 363 estudiantes afectados, llegando a la conclusión que producto de dicha coyuntura, solo el 34\% terminará sus estudios en sus universidades bajo las condiciones en las que iniciaron, el 27\% deberá migrar a otras universidades dentro y fuera de la región, el 17\% dejarán de formarse profesionalmente y un $21 \%$ se encuentra en total incertidumbre.

Palabras Clave: Licenciamiento institucional; Efectos sociales; Deserción educativa; Migración educativa

\begin{abstract}
The article presents the descriptive analysis of the social effects of the University Licensing process on students from non-licensed universities in the Cusco region, under the categories of dropout and educational migration, this research with a mixed focus, descriptive level and nonexperimental design - cross-sectional, I apply a survey to 363 affected students, reaching the conclusion that, as a result of this situation, only $34 \%$ of this population will finish their studies at their universities under the conditions in which they started, $27 \%$ must migrate to other universities within and outside the region, $17 \%$ of students will stop training professionally and $21 \%$ are in total uncertainty.
\end{abstract}

Keywords: Institutional licensing, social effects, educational desertion, educational migration 


\section{INTRODUCCIÓN}

La concepción de universidad se remonta a la primera acepción de academia, nombre que recibía la escuela fundada por Platón, la cual trascendía el entorno físico de reunión y se fundamentaba en el profundo deseo de saber la verdad. Este análisis filosófico infiere que la universidad refleja el espíritu humano y en ella se hace realidad "la theoria, es decir la actitud contemplativa" 1 (Frank, 2009, p. 17).

Ya en el siglo XIX, en Inglaterra y otros países de Europa y Estados Unidos, surgen diversas universidades bajo el modelo humanista de Humboldt que incorporaban a la definición de universidad funciones sustantivas como ciencia e investigación (Medina Coronado, 2018, p. 706), situación que se replicó en América Latina. Desde ese entonces hasta la actualidad, el término universidad ha ido evolucionando, de manera que, en la conceptualización de universidad del siglo XXI, incorpora aspectos como responsabilidad social, tecnologías de información, cooperación, entre otros.

En el desarrollo histórico de la universidad se han generado diferentes modelos o concepciones que articulan distintas

\footnotetext{
${ }^{1}$ Teoría: de latín theoria y este "del griego theorein, que significa mirar atentamente, contemplar" (Frank, 2009).
}

visiones sobre sus fines y funciones, su organización y su relación entre estudiante, ciencia y Estado. Las transformaciones vividas por la universidad en las últimas décadas han generado que no se tenga un modelo definido. El afán de la profesionalización, la hegemonía de la razón científica moderna, el excesivo cultivo de la persona y los desafíos paradigmáticos, han impregnado en el currículo educativo aspectos que no se han logrado integrar de manera armónica (Dreifuss Escarate \& Vélez Valcárcel, 2010, p. 67).

La universidad en el Perú ha sufrido diversas transformaciones, algunas relacionadas al fenómeno de expansión, las cuales han impactado directamente en la teleología universitaria; además, cuestionando el papel de la universidad en el orden social, desde una perspectiva política y filosófica, el concepto de desarrollo vinculado a la masificación pudo deslindarse del utilitarismo asociado a la teoría del capital humano. El explosivo crecimiento se debió a la decisión del Estado peruano que, en 1996, liberalizó el mercado educativo universitario, lo cual generó un desequilibrio entre la demanda y las necesidades del mercado laboral y también la postergación 
de la investigación en la formación universitaria (Cuenca, 2015, p. 10).

Si bien los argumentos de la liberalización fueron positivos -como la ampliación de la oferta educativa y democratizar la educación-, pensar que el mercado sería un regulador efectivo de calidad fue un error, ya que la oferta de instituciones creció sin mayor regulación y las universidades fueron politizadas $\mathrm{y}$ vistas como inversiones millonarias, dejando de lado la calidad. Todo ello trajo consigo que las universidades peruanas no figurasen en ranking mundiales ni latinos, que el volumen y calidad de investigación fuesen incipientes y que existiesen bajos porcentajes de empleabilidad de egresados universitarios.

Es en ese contexto que, a mediados de 2013, se inicia la reforma educativa y mediante la Ley Universitaria $N^{\circ} 30220$ se pretenden resolver los problemas de calidad generados por la masificación de oferta educativa. La Ley Universitaria conceptualiza la universidad como "una comunidad académica orientada a la investigación y a la docencia, que brinda una formación humanista, científica y tecnológica con una clara conciencia de nuestro país como realidad multicultural" (Ley Nº 302202014 , art. 3). En ese marco, la autoridad es la encargada de proveer herramientas materiales, tecnológicas y gnoseológicas a toda la comunidad universitaria, garantizando que, a través del trabajo armónico e integrado de sus estamentos, se logre calidad educativa en cada una de sus funciones sustantivas: formación profesional, investigación, extensión cultural, proyección social, educación continua y la contribución al desarrollo humano.

El ingreso de la Ley Universitaria trajo consigo un sinnúmero de debates, ya que desnudó la difícil realidad educativa universitaria que tenía el país, "el descuido del Estado con la universidad pública, la heterogénea calidad de oferta privada, el débil movimiento estudiantil, los impresionantes sueldos de autoridades universitarias, la influencia de la clase política en decisiones educativas 'lobistas', el limbo legal en el que operaba la Asamblea Nacional de Rectores ANR, la incapacidad de gasto de las universidades públicas" (Cuenca, 2015, p. 13)

entre otros efectos de las décadas de inoperancia de la autoridad del sector educativo superior en el país.

La Superintendencia Nacional de Educación Superior Universitaria (SUNEDU), actualmente tiene a cargo salvaguardar el cumplimiento de la Ley Universitaria, regulando con ello la calidad educativa. SUNEDU tiene como finalidad proteger el derecho de los jóvenes a recibir una educación universitaria de calidad y, de esta 
manera, mejorar sus competencias profesionales. A partir de enero de 2015, es la responsable del otorgamiento del licenciamiento institucional que comprende la verificación del cumplimiento de condiciones básicas de calidad en las universidades del país. Al constatar el cumplimiento de dichas condiciones, otorga la autorización de funcionamiento que es temporal y renovable. Las condiciones básicas de calidad están referidas a siete aspectos:

Tabla 1

Condiciones básicas de calidad

Condiciones básicas de calidad

1. La existencia de objetivos académicos, grados y títulos a otorgar y planes de estudio

2. Previsiones económicas y financieras de la universidad a crearse, compatible con los fines propuestos en sus instrumentos de planteamiento

3. Infraestructura y equipamiento adecuados al cumplimiento de sus funciones (bibliotecas, laboratorios, entre otros)

4. Líneas de investigación a ser desarrolladas

5. Verificación de la disponibilidad de personal docente calificado con no menos del 25 $\%$ de docentes a tiempo completo

6. Verificación de los servicios educacionales complementarios básicos (servicio médico, social, psicopedagógico deportivo, entre otros)

7. Existencia de mecanismos de mediación e inserción laboral (bolsa de trabajo u otros) Extraído de Ley Universitaria, Ley $\mathrm{N}^{\circ}$ 30220, artículo 28.

Entre el 15 de diciembre de 2015 y el 15 de diciembre de 2017, 141 universidades y 04 Escuelas de posgrado presentaron su solicitud de licenciamiento institucional ante la SUNEDU y, hasta el 14 de marzo de 2020, 91 universidades y 2 escuelas de posgrado fueron licenciadas y 43 universidades y 2 escuelas de posgrado tienen la licencia denegada. Esto ha generado que la oferta de educación universitaria a nivel nacional haya sufrido un decremento producto de este proceso de licenciamiento.

Cusco, hasta el año 2019, tenía 6 universidades operando en la región, cuya oferta educativa se llevaba a cabo de manera presencial y atendía a la demanda de educación superior de los jóvenes cusqueños. Es innegable que la regulación y el estricto cumplimiento de parámetros de calidad educativa representan para el país un gran paso como sociedad. Sin embargo, el efecto que se ha producido en las regiones ha sido distinto al de la capital, donde la oferta educativa heterogénea es mayor. Para el caso, en la Ley Universitaria no se han encontrado acciones de contingencia que prevean la situación de incertidumbre de los afectados. Es así que se han puesto en evidencia grandes falencias en cuanto a la planeación y prospectiva, siendo la 
población afectada en su mayoría estudiantes. De las 6 universidades que operaban en la región del Cusco al 2019, 3 de ellas obtuvieron el licenciamiento:

Tabla 2

Estado del licenciamiento en universidades del Cusco

\begin{tabular}{|c|c|c|c|c|}
\hline Universidad & $\begin{array}{l}\text { Estado del } \\
\text { proceso }\end{array}$ & $\begin{array}{l}\text { Fecha de } \\
\text { resolución }\end{array}$ & $\begin{array}{c}\text { Resolución de } \\
\text { Licenciamiento }\end{array}$ & $\begin{array}{l}\text { Tipo de } \\
\text { gestión }\end{array}$ \\
\hline $\begin{array}{l}\text { Universidad Andina } \\
\text { del Cusco }\end{array}$ & Licenciada & $23 / 12 / 2017$ & $\begin{array}{l}\text { Resolución del Consejo } \\
\text { Directivo N074-2017- } \\
\text { SUNEDU/CD }\end{array}$ & Privada \\
\hline $\begin{array}{l}\text { Universidad } \\
\text { Nacional San } \\
\text { Antonio Abad del } \\
\text { Cusco }\end{array}$ & Licenciada & $04 / 05 / 2019$ & $\begin{array}{l}\text { Resolución del Consejo } \\
\text { Directivo N056-2019- } \\
\text { SUNEDU/CD }\end{array}$ & Pública \\
\hline $\begin{array}{l}\text { Universidad Global } \\
\text { del Cusco }\end{array}$ & $\begin{array}{l}\text { Licencia } \\
\text { denegada }\end{array}$ & $24 / 09 / 2019$ & $\begin{array}{c}\text { Resolución del Consejo } \\
\text { Directivo N¹23-2019- } \\
\text { SUNEDU/CD }\end{array}$ & Privada \\
\hline $\begin{array}{l}\text { Universidad Peruana } \\
\text { Austral del Cusco }\end{array}$ & $\begin{array}{l}\text { Licencia } \\
\text { denegada }\end{array}$ & $08 / 11 / 2019$ & $\begin{array}{c}\text { Resolución del Consejo } \\
\text { Directivo N¹43-2019- } \\
\text { SUNEDU/CD }\end{array}$ & Privada \\
\hline $\begin{array}{l}\text { Universidad Alas } \\
\text { Peruanas (filial } \\
\text { Cusco) }\end{array}$ & $\begin{array}{l}\text { Licencia } \\
\text { denegada }\end{array}$ & $26 / 12 / 2019$ & $\begin{array}{c}\text { Resolución del Consejo } \\
\text { Directivo N¹72-2019- } \\
\text { SUNEDU/CD }\end{array}$ & Privada \\
\hline $\begin{array}{l}\text { Universidad } \\
\text { Tecnológica de los } \\
\text { Andes (filial Cusco) }\end{array}$ & Licenciada & $29 / 02 / 2020$ & $\begin{array}{l}\text { Resolución del Consejo } \\
\text { Directivo N031-2020- } \\
\text { SUNEDU/CD }\end{array}$ & Privada \\
\hline
\end{tabular}

Según ordena la norma, las universidades no licenciadas tendrán dos años para terminar de operar y proceder a su cierre y dos años más para tramites de titulación de sus estudiantes. Esta situación obliga a analizar cuáles son los efectos sociales del proceso de Licenciamiento Universitario en los estudiantes de universidades no licenciadas en la región Cusco, siendo necesario observar el nivel de deserción y migración educativa universitaria.
En la revisión y análisis del estado del arte, se encontraron investigaciones que permitieron observar al objeto del estudio, entre ellas la investigación de Gallegos (2017), en la que se analiza el proceso de licenciamiento y su impacto en la educación superior universitaria del Perú, a partir de la entrada en vigencia de la Ley Universitaria (Ley $\mathrm{N}^{\circ}$ 30220) en julio de 2014. Dicha investigación muestra el proceso administrativo para la implementación de la norma, evidenciando la existencia de vacíos 
en cuanto a la programación de presupuesto para las universidades públicas, que debían invertir en las condiciones básicas de calidad de manera planificada.

Tupayachi \& López (2018) desarrollan una visión propositiva acerca de las nuevas políticas que las universidades interculturales del Perú podrían implementar para obtener el licenciamiento y, por ende, brindar una educación universitaria de calidad. Concluyen que las condiciones básicas de calidad no estaban fundamentadas en la realidad de todas las universidades públicas y privadas del país.

En la investigación de Angulo, Angulo, Coronel \& Espinoza (2016) se proponen estrategias para afrontar el proceso de licenciamiento, sincretizando tanto la Ley Universitaria como el ISO $9001 \mathrm{y}$ desarrollando actividades tangibles orientadas al mejoramiento continuo.

Estas investigaciones científicas brindan aportes dirigidos al proceso de licenciamiento y a las acciones que como universidades se deben llevar a cabo para obtener dicho licenciamiento; sin embargo, los estudios acerca de los efectos que este proceso genera en la población estudiantil son mínimos y tienen como principal fuente de información investigaciones periodísticas.
A nivel de América Latina, el estudio de Gonzales (2005) hace un análisis de la evaluación y acreditación en los sistemas nacionales universitarios de Argentina, Bolivia, Brasil, Chile, Colombia, Costa Rica, Ecuador, México, Paraguay, Perú, República Dominicana, Uruguay y Venezuela, concluyendo que existe una reluctancia a la implementación de cambios, ya que el crecimiento, la diversificación de la oferta educativa y la preocupación de los gobiernos por garantizar un servicio educativo de calidad, tienen limitaciones y diferencias entre lo público y privado.

En el Perú, el artículo publicado por el Vicerrectorado Académico de la Pontifica Universidad Católica del Perú (2019) habla acerca de los avances y efectos residuales del licenciamiento de universidades y menciona las protestas a nivel nacional por parte de los estudiantes de universidades no licenciadas, exponiendo la existencia de aspectos no contemplados en la normas que afectan a los estudiantes y demás miembros de las universidades. Por una parte, el incumplimiento de las universidades para facilitar los procesos de traslados de los estudiantes afectados y, por otra parte, que, pese a que los estudiantes ya habían escogido mecanismos para continuar sus estudios, la SUNEDU, a abril de 2019, aún no había terminado la difusión y orientación a estudiantes. Se mencionan también diferentes medidas que, producto de la 
implementación o mejora de las condiciones básicas de calidad en las universidades, han tenido efectos positivos, como el fortalecimiento de la carrera docente, ya que ahora se cuenta con más docentes a tiempo completo, y la mayor inversión de proyectos de investigación, entre otros.

El estudio de estos antecedentes permite contextualizar las diferentes palabras claves necesarias para entender el presente artículo. El efecto social, según la Real Academia, puede verse como un cambio en el resultado de un proceso. En este caso, el cambio que ocasiona el proceso de licenciamiento. Según la Ley Universitaria $\mathrm{N}^{\circ} 30220$, el licenciamiento universitario es un procedimiento que se encarga de comprobar la calidad educativa que tenga la educación superior en las universidades peruanas. Dentro de los efectos sociales de este proceso se analizará específicamente la "deserción educativa", que consiste en la situación de abandono o separación por parte de estudiante en su formación profesional universitaria y también, la "migración educativa", que implica la movilización o desplazamiento de esta población estudiantil a causa de su situación académica actual.

\section{Método}

La investigación tiene un enfoque mixto, de tipo básica, con un alcance de investigación descriptivo y un diseño de investigación no experimental de corte transversal. La población de estudio está integrada por los estudiantes matriculados en el semestre 2019-II, de las universidades no licenciadas en el Cusco, que hacen un total de 6701 estudiantes, en sus diferentes programas académicos, bajo el siguiente detalle:

\section{Tabla 3}

Población de estudio: Estudiantes de universidades no licenciadas en la región del Cusco

\begin{tabular}{cc}
\hline Universidad no & Programas \\
$\begin{array}{c}\text { Licenciada en la } \\
\text { región }\end{array}$ & $\begin{array}{c}\text { Total de } \\
\text { estudiantes }\end{array}$ \\
\hline
\end{tabular}

Ciencias Contables y Finanzas

Universidad Austral del Cusco

Universidad Alas Peruanas Filial Cusco

$$
\text { Ingeniería de Sistema y Seguridad Informática }
$$
Economía y Negocios Internacionales 889

Turismo Ingeniería Civil Ingeniería Ambiental 5221 


\section{Ciencias Contables y Financieras}

Derecho

Estomatología

Psicología Humana

Administración y Negocios Globales

Universidad Global del Cusco

Administración de negocios Turísticos

Ingeniería en tecnologías de la Información y

comunicación

Total

6701

Extraído de los informes técnicos publicados por la Superintendencia Nacional de Educación Superior Universitaria (SUNEDU) https://www.sunedu.gob.pe/avances-licenciamiento/

La muestra fue de 363 estudiantes, los cuales se seleccionaron mediante el muestreo probabilístico aleatorio simple. Se utilizó la técnica de recolección de datos "encuesta", y mediante un cuestionario de 11 preguntas, dividido en 3 categorías: caracterización de la población de estudio, deserción y migración educativa. De igual forma, se utilizó la guía de entrevista estructurada, aplicada a representantes estudiantiles. El recojo, procesamiento y análisis de datos se llevó a cabo en los meses de marzo, abril y mayo de 2020, mediante plataformas digitales como Google Drive y Google Meet.

\section{Resultados}

Después del proceso de interpretación de datos, se obtuvo como resultado que un 40 $\%$ de los encuestados tienen edades entre los 18 y 23 años, otro $40 \%$ tiene entre 24 y 28 años y solo un $19 \%$ tiene edades mayores a 29 años. Así, a la población afectada queda caracterizada como una población estudiantil joven entre 18 y 28 años.

Los resultados hallados, correspondientes al OG1: Determinar cuáles son los efectos sociales del proceso de Licenciamiento Universitario en los estudiantes de universidades no licenciadas en la región Cusco, están relacionados con los resultados de la Tabla 4, en la que se puede apreciar que el $66 \%$ de los estudiantes debe tomar diferentes alternativas para afrontar su futuro académico profesional, ya que solo el $34 \%$ manifiesta que terminara sus estudios en sus universidades. Actualmente, el $27 \%$ tomo la decisión de trasladarse a otras universidades dentro y fuera de la región, un $1 \%$ decidió trasladarse a institutos superiores, un $17 \%$ manifiesta que dejara de estudiar y un preocupante $21 \%$ indica que no han decidido que hará con respecto a su educación. 
Tabla 4

Acciones con respecto a su educación

\begin{tabular}{|c|c|c|}
\hline Acciones que tomara con respecto a su educación & Frecuencia & Porcentaje \\
\hline Aún no lo ha decidido & 77 & $21 \%$ \\
\hline Dejar de estudiar por el momento & 61 & $17 \%$ \\
\hline Trasladarse a estudiar a un instituto superior & 2 & $1 \%$ \\
\hline Trasladarme a una universidad de la región & 70 & $19 \%$ \\
\hline Trasladarme a una universidad de otra región & 29 & $8 \%$ \\
\hline $\begin{array}{l}\text { Terminar mis estudios en la universidad por el plazo que dure } \\
\text { su cierre }\end{array}$ & 124 & $34 \%$ \\
\hline Total & 363 & $100 \%$ \\
\hline
\end{tabular}

Extraído de la encuesta a estudiantes mediante el Google Drive

Estos resultados se respaldan con el análisis hecho de la entrevista a los representantes estudiantiles de universidades no licenciadas, los cuales se muestran en la Tabla 5.

Tabla 5

Análisis de la entrevista

Aspectos Análisis $\quad$ Conclusiones

\begin{tabular}{clll}
\hline Difusión de & Los entrevistados manifiestan & La información tanto & de \\
información & no haber recibido información & SUNEDU como de la \\
(continuidad de & clara y precisa por parte de & universidad no fue clara ni \\
estudios y convenios) & SUNEDU y su universidad. & precisa. & \\
& Los entrevistados indicaron que & De acuerdo al semestre, algunos \\
& los estudiantes de semestres & estudiantes se cambiaron de \\
Situación académica de & inferiores con posibilidades & universidad, algunos desertaron \\
estudiantes (menores a & económicas se cambiaron, pero por no tener medios \\
VI semestre y mayores & algunos dejaron de estudiar. & económicos y solo los \\
a VI semestre) & Los de semestres superiores, estudiantes en semestres \\
& están terminando en la & superiores continúan en la \\
& universidad. & universidad. & \\
Los entrevistados manifestaron & La oferta educativa actual tiene \\
Situación de estudiantes & que las tarifas de otras & tarifas por encima al pago que \\
que migraron a otras & universidades están por encima & hacían antes y la convalidación, \\
universidades (tarifas & de lo que ellos pagaban que el & al ser potestad de la universidad \\
de estudio y & tema de la convalidación era & receptora, en su mayoría no \\
convalidaciones) & independiente a cada & lograron convalidar muchas de \\
& universidad receptora. En & sus asignaturas. & \\
\hline
\end{tabular}


muchos casos, no procedió la

convalidación.

Sentir de los estudiantes afectados (efecto en sus relaciones familiares $y$ amicales)

Los entrevistados expusieron sentimientos de desamparo, desilusión, depresión, ya que sus planes se truncaron. Este sentimiento también lo tienen sus familias.

Los estudiantes afectados tienen sentimientos de desamparo, desilusión y depresión que afecto a sus hogares, sobre todo aquellos que se auto sostienen

Extraído de la entrevista a representantes estudiantiles mediante el Google Hangouts Meet

Algunos efectos sociales importantes de remarcar son aquellos que se refieren al sentimiento de desamparo por parte de los estudiantes en cuanto a su futuro académico, ya que algunos deberán comenzar nuevamente sus carreras profesionales por la no convalidación de muchas asignaturas. Esto es una demostración de la falta de información con respecto a los convenios con otras universidades. Como indica SUNEDU, esta problemática se agrava debido a que la población estudiantil afectada, en su mayoría, son jóvenes que se auto sostienen y que por razones económicas no pueden cubrir las tarifas solicitadas por la oferta educativa actual, lo que los motiva a la deserción.

Los resultados hallados, correspondientes al OE1: Identificar cuál es el nivel de deserción educativa universitaria a raíz del proceso de Licenciamiento Universitario en la región Cusco, se basan, primero, en el entendimiento de que la realidad de los estudiantes de semestre inferiores al VI, es diferente a estudiantes en semestres superiores, puesto que el proceso de cierre de las universidades tiene un plazo de 2 años, lo cual permitiría a esos estudiantes de semestres superiores acabar sus estudios en su universidad y luego tramitar su titulación.

En la Tabla 6, se puede apreciar la diferencia de estos dos contextos, en los que el nivel de deserción, en el caso de estudiantes de I, II, III, IV, V y VI semestres, es de $42 \%$, lo cual tiene relación a aspectos económicos y de tiempo. Por otro lado, en el caso de estudiantes de VII, VIII, IX y $\mathrm{X}$ el porcentaje de deserción es de $10 \%$. En ambas poblaciones estudiantiles, el porcentaje de estudiantes que no han decidido qué hacer con respecto a su educación, supera el $20 \%$.

\section{Tabla 6}

Acciones que tomará con respecto a su educación según estudiantes por semestre de estudio

\begin{tabular}{|c|c|c|c|}
\hline Estudiantes & Valor & Acciones que tomara & Valor \\
\hline $\begin{array}{c}\text { por } \\
\text { semestres } \\
\text { de estudio }\end{array}$ & Frecuencia Porcentaje & $\begin{array}{c}\text { con respecto a su } \\
\text { educación }\end{array}$ & Frecuencia Porcentaje \\
\hline
\end{tabular}




\begin{tabular}{|c|c|c|c|c|c|}
\hline \multirow{4}{*}{$\begin{array}{l}\text { Estudiantes } \\
\text { de I, II, III, } \\
\text { IV, V y VI } \\
\text { semestres }\end{array}$} & \multirow[t]{4}{*}{73} & \multirow[t]{4}{*}{$20 \%$} & Aun no lo han decidido & 17 & $23 \%$ \\
\hline & & & $\begin{array}{l}\text { Dejar de estudiar por el } \\
\text { momento }\end{array}$ & 31 & $42 \%$ \\
\hline & & & $\begin{array}{l}\text { Trasladarme a otra } \\
\text { universidad de la región }\end{array}$ & 18 & $25 \%$ \\
\hline & & & $\begin{array}{l}\text { Trasladarme a otra } \\
\text { universidad en otra } \\
\text { región }\end{array}$ & 7 & $10 \%$ \\
\hline \multirow{6}{*}{$\begin{array}{l}\text { Estudiantes } \\
\text { de VII, } \\
\text { VIII, IX y } \\
\text { X }\end{array}$} & \multirow[t]{6}{*}{290} & \multirow[t]{6}{*}{$80 \%$} & Aun no lo han decidido & 60 & $21 \%$ \\
\hline & & & $\begin{array}{l}\text { Dejar de estudiar por el } \\
\text { momento }\end{array}$ & 30 & $10 \%$ \\
\hline & & & $\begin{array}{l}\text { Trasladarme a estudiar } \\
\text { en un instituto superior }\end{array}$ & 2 & $1 \%$ \\
\hline & & & $\begin{array}{c}\text { Trasladarme a otra } \\
\text { universidad de la región }\end{array}$ & 52 & $18 \%$ \\
\hline & & & $\begin{array}{l}\text { Trasladarme a otra } \\
\text { universidad en otra } \\
\text { región }\end{array}$ & 22 & $8 \%$ \\
\hline & & & $\begin{array}{l}\text { Terminar mis estudios en } \\
\text { la Universidad por el } \\
\text { plazo que dure su cierre }\end{array}$ & 124 & $43 \%$ \\
\hline Total & 363 & $100 \%$ & & 363 & \\
\hline
\end{tabular}

Extraído de la encuesta a estudiantes mediante el Google Drive

En cuanto al OE2: Identificar cuál es el nivel de migración educativa universitaria a raíz del proceso de Licenciamiento Universitario en la región Cusco, la Tabla 6 también hace referencia a este aspecto. Así, la población estudiantil de I a VI semestre alcanza un porcentaje de migración del $35 \%$, de los que el $25 \%$ realizó traslados dentro de la región Cusco, pero el $10 \%$ migró a otras regiones, siendo el principal destino Arequipa, en segundo lugar, Apurímac y en tercer lugar Lima.

En el caso de la población estudiantil de VII a X semestre, el porcentaje de migración es de $26 \%$, de los cuales el $18 \%$ migrará a universidades de la región y un $8 \%$ migrará a universidades de otras regiones. Se podría considerar que, pese a que la Ley Universitaria garantiza la culminación de sus estudios en sus universidades de origen, prefieren realizar sus traslados.

\section{DISCUSIÓN}

La investigación analizó los efectos sociales del proceso de licenciamiento en estudiantes de universidades no licenciadas, bajo los criterios de nivel de deserción y nivel de migración. En cuanto a la validez interna, es necesario precisar que los resultados muestran la percepción del problema por parte del segmento estudiantil afectado y que la triangulación con la autoridad universitaria y la SUNEDU permitiría tener 
conclusiones más certeras, siendo esta la única limitación para un desarrollo metodológico más efectivo. En cuanto a la validez externa, el método usado para la investigación puede ser replicado en otras regiones del país, con la finalidad de mostrar de manera más real los efectos sociales del proceso de licenciamiento en todas las regiones, lo que permitiría proponer recursos particulares a cada realidad.

Los resultados obtenidos guardan relación con la investigación de Gonzales (2005), ya que en el contexto estudiado también se aprecian fuertes diferencias entre la oferta educativa pública y privada. Así pues, la tarifa educativa de la oferta privada existente hace inalcanzables los traslados de estudiantes afectados. Este es un aspecto de índole social no considerado por la Ley Universitaria ni la autoridad pertinente, que encuentra otra aproximación con el artículo publicado por el Vicerrectorado Académico de la Pontifica Universidad Católica del Perú (2019), en el que se menciona la existencia de efectos residuales del licenciamiento universitario que desnudan el desamparo de información y atención a los estudiantes afectados, situación que es similar a la que se encuentra en la región Cusco. Es indudable que los efectos de la implementación de condiciones básicas de calidad en la educación universitaria garantizan una mejora integral del sistema universitario y las universidades no licenciadas deberán responder y actuar como corresponde según la ley. Esta coyuntura abre nuevos horizontes de investigación, entre ellos los efectos sociales de los estudiantes de universidades licenciadas, la perspectiva de la sociedad civil ante este panorama y la posición del mercado laboral a través de cálculos de demanda profesional, entre otros.

\section{CONCLUSIONES}

Los estudiantes de universidades no licenciadas en la región Cusco, afectados por el proceso de Licenciamiento Universitario, son una población juvenil entre 18 y 28 años de edad que mayoritariamente se auto sostienen y tienen posibilidades económicas limitadas. Producto de esta coyuntura, solo el $34 \%$ de esta población terminará sus estudios en sus universidades bajo las condiciones en las que iniciaron, el $27 \%$ deberá migrar a otras universidades dentro y fuera de la región y el $1 \%$ se trasladará a institutos superiores. En cuanto a la población estudiantil que se siente desamparada por sus autoridades, $17 \%$ de estudiantes dejarán de formarse profesionalmente y un $21 \%$ se encuentra en total incertidumbre por factores de índole económico en relación a la diferencia de tarifas educativas y dificultades de convalidación, entre otros.

El nivel de deserción educativa a raíz del proceso de licenciamiento universitario en la 
región Cusco, en estudiantes del I al VI semestre, es de $42 \%$ y el nivel de deserción de los estudiantes entre el VII y X semestre es del $10 \%$.

El nivel de migración educativa a raíz del proceso de licenciamiento universitario en la región Cusco, en la población estudiantil entre I y VI semestre alcanza el $35 \%$, compuesto por el $25 \%$ de traslados dentro de la región y un $10 \%$ de migración a otras regiones. En el caso de la población estudiantil entre VII y X semestre, el porcentaje de migración es de $26 \%$, de los cuales el $18 \%$ migrará a universidades de la región y un $8 \%$ migrará a universidades de otras regiones, siendo los principales destinos Arequipa, Apurímac y Lima.

\section{REFERENCIAS BIBLIOGRÁFICAS}

Angulo Herrera, P., Angulo Alvarado, P., Coronel Capacyachi, E., \& Espinoza Blanco, J. (2016). Consideraciones y estrategias para la implementación de un sistema de gestión de calidad ISO 9001 en el marco del Licenciamiento y la Acreditación de la Educación Superior Universitaria en el Perú. Revista Apuntes de Ciencias \& Sociedad vol. 6.

Cuenca, R. (2015). La educación universitaria en el Perú. Democracia, expansión y desigualdad. Lima,
Perú: Instituto de Estudios Peruanos IEP.

Dreifuss Escarate, D., \& Velez Valcarcel, O. (2010). El poder de educar, una mirada al vínculo pedagógico . LIma, Perú: Universidad Peruana de Ciencias Aplicadas (UPC).

Frank, J. F. (2009). La filosofía y la universidad. Una leccion de antropología. Humanidades, VIII(1), 10-17.

Gallegos, A. (2017). Educación superior y licenciamiento: El caso de las universidades del Perú. Revista Espacios, 38(60), 35.

Gonzales, L. (2005). El impacto del proceso de evaluación y acreditación en las universidades de América Latina. CINDA - IESALC/UNESCO.

Ley Universitaria, Ley N³0220 (2014). Lima, Perú: El Peruano.

Medina Coronado, D. (2018). El rol de las universidades peruanas frente a la investigación y el desarrollo tecnológico. Propósitos y Representaciones 6, 703-737.

Pontificia Universidad Católica del Perú (Abril de 2019). Noticias y eventos. Obtenido de https://vicerrectorado.pucp.edu.pe/a cademico/noticias/avances-efectos- 
residuales-del-licenciamiento-

universidades/

Superintendencia Nacional de Educación

Superior Universitaria (SUNEDU)

(Abril de 2020). Página Oficial

SUNEDU. Obtenido de

https://www.sunedu.gob.pe/avances

-licenciamiento/

Tupayachi Díaz, C., \& López Vega, M. (2018). Implementación de Nuevas Políticas para SUNEDU en el proceso de licenciamiento de universidades interculturales del Perú [Tesis de Maestría, Universidad César Vallejo]. Perú: Escuela de Postgrado-Universidad César Vallejo. 